\title{
The Role of Mediators in Reducing Antepartum Depressive Symptoms in Rural Low-Income Women Receiving a Culturally Tailored Cognitive Behavioral Intervention
}

\author{
D. Elizabeth Jesse, PhD, CNM, Hui Bian, PhD, CHES, Elizabeth C. Banks, PhD, LMFT, \\ Bradley N. Gaynes, MD, MPH, Steve D. Hollon, PhD, and Edward R. Newton, MD
}

\section{Introduction}

Depending on the population studied and the screening instrument used, 10-66\% of pregnant women experience antepartum depressive symptoms that indicate risk for antepartum depression (Gaynes et al., 2005; Giurgescu, Engeland, \& Templin, 2015; Gourounti, Karapanou, Karpathiotaki, \& Vaslamatzis, 2015; Jallo et al., 2015; Jesse, Kim, \& Herndon, 2014). In two national samples (Fellenzer \& Cibua, 2014; Vesga-López et al., 2008), $8 \%-14.5 \%$ of women met criteria for antepartum depression, a major depressive disorder in pregnancy. In previous studies (Jesse et al., 2014; Jesse \& Swanson, 2007), a third of rural low-income women were found to be at risk for antepartum depression, especially if they experienced high stress. Pregnant women with antepartum depressive symptoms (APDS) or antepartum depression (APD) may experience a stressful, sad, and joyless pregnancy, greater psychosocial and behavioral risk (Jesse et al., 2014; Smedberg, Lupatelli, Mardby, Overland, \& Nordeng, 2015), higher rates of preterm birth (Accortt \& Schetter, 2014; Giurgescu et al., 2015) and low-birth weight infants (Giurgescu et al., 2015; Tomita, Labys, \& Burns, 2015), more frequent postpartum depression (Witt et al., 2011), and even death by suicide (Palladino, Singh, Campbell, Flynn, \& Gold, 2011).

Despite growing recognition of the consequences of this public health problem, women at risk for APD often go undetected and untreated (Ko, Farr, Dietz, \& Robbins, 2012). The authors of an earlier study (Jesse, Dolbier, \& Blanchard, 2008) reported that in a similar population of low-income and rural minority women few keep a referral appointment to a mental health provider because of inadequate transportation, language barriers, stigma, and fear of being labeled an "unfit" mother (Jesse et al., 2008).

Cognitive behavioral interventions (CBIs) have demonstrated efficacy in reducing depressive symptoms in the general adult population (Linde et al., 2015) but only one randomized controlled clinical trial (Jesse et al., 2015), has shown the preliminary efficacy of a culturally tailored CBI for reducing APDS in rural low-income and minority women with mild to moderate depressive symptoms. That trial showed similar results for African-American women at high-risk for depression. No existing research literature explained the mechanisms by which a CBI reduces APDS in women or used a formal theoretical model to explain the mechanisms. This research will add to the existing knowledge base by (1) examining specific content not currently found in the literature and (2) explicating the mechanisms of 
this culturally tailored CBI to strengthen intervention models that address the needs of women at risk for antepartum depression.

The purpose of this paper is to determine the mechanisms of a culturally-tailored CBI, Insight-Plus, tested in a RCT which has been reported elsewhere (Jesse et al., 2015). The CBI was guided by Jesse's bio-psychosocial-spiritual midrange theory of risk for antepartum depression described earlier (Jesse et al., 2014; Jesse \& Swanson, 2007), augmented by aspects of Gordon, Matwychuk, Sachs, and Canedy's (1988) cognitive-behavioral program for non-pregnant women and Beck's cognitive model of depression for adults (Beck, 1967, 1976). The model of this study's theoretical framework is displayed in Figure 1. We hypothesized that (1) Insight-Plus would reduce levels of stress and negative thinking, enhance levels of self-esteem, and increase social support; and (2) controlling for race, these effects would mediate the effects in reducing antepartum depressive symptoms from baseline to 1-month follow-up.

\section{Methods}

\section{Design}

This was a cross-sectional research design and second analysis of primary data from a randomized controlled clinical trial. Ethical approval for this study was obtained from the East Carolina University's Institutional Review Board.

\section{Participants}

The analysis included 110 rural low-income African-American, Caucasian, and Hispanic pregnant women in the RCT who completed all measures at baseline (T1) and a 1-month follow-up (T3) interview. All participants in the RCT were enrolled in prenatal care at a local health department and an affiliated regional perinatal center prenatal care setting. All were at least 18 years old, gestationally between 6 and 30 weeks, self-identified as African American, Caucasian, or Hispanic and were low income, as determined by Medicaid or WIC (Special Supplemental Nutrition Program for Women, Infants, and Children) eligibility criteria. All could read at least at a $4^{\text {th }}$ grade level. Women with miscarriage or fetal demise, schizophrenia or bipolar diagnosis, current treatment for depression, a high-risk pregnancy diagnosis, suicidal ideation that included a plan, or have a medical condition that could explain depression, such as hypothyroidism were excluded. Women who scored 4 or above on the Edinburgh Postnatal Depression Scale (EPDS) (Cox, Holden, \& Sagovsky, 1987) were enrolled in the study and randomly assigned to the CBI or treatment-as-usual after giving informed consent. Antepartum depressive symptoms, not APD, were the focus of the intervention.

Immediately after giving informed consent, participants completed a baseline (T1) interview. Each woman received a $\$ 20$ gift card for the baseline interview (T1) and, $\$ 50$ for each of the follow-up interviews. A time 2 interview (T2) occurred after the last CBI session and a final interview (T3) was scheduled 1-month after the last session. An interpreter was available for all interviews and sessions with Spanish speaking women. 


\section{Culturally Tailored Cognitive Behavioral Intervention (CBI)}

The problem-oriented CBI was designed to help women with antepartum depression symptoms manage stress, identify and replace negative thoughts, set goals, break tasks into smaller components, and solve problems (Jesse et al., 2015). The women were also encouraged to increase positive events and activities and seek out social support.

A master's prepared mental health professional facilitated the CBI group sessions. A "Resource Mom" support staff contacted the women weekly for a booster session, encouraged them to continue with their homework, supported them through personal problems, reminded them of their next session or interview, and arranged transportation and childcare as needed.

Treatment-as-usual consisted of standard treatment by social workers or pregnancy care managers at the clinical sites. All women were assessed by nurses and the pregnancy care managers for risk of depression and were referred to community resources as needed.

\section{Study Measures}

Demographics-At baseline, we asked the women for socio-demographic data including age, ethnicity/race, type of insurance, education, partner status, gravida, and parity.

Primary outcome measures-The primary outcome measure for antepartum depression symptoms was the 10-item Edinburgh Postnatal Depression Scale (EPDS) (Cox et al., 1987) used to measure antepartum depressive symptoms within the past 7 days. Each item is on a 4-point scale (0-3) and total scores range from 0 to 30 . For example, on the item, "I have been able to laugh and see the funny side of things," answers range from, "as much as I always could (0)" to "not at all (3). " and on the item, "I have felt sad and miserable," answers range from "yes, most of the time (3)" to "no, not at all (0)." The scale takes less than 5 minutes to administer and is at the $3^{\text {rd }}$ grade readability level. Cronbach's alpha for the EPDS scale is .84 .

The 21-item Beck Depression Inventory (BD-II) (Beck, Brown, \& Steer, 1996) was used to measure antepartum depressive symptom severity within the past 7 days. The BDI-II uses a 4-point scale ranging from 0-3; item responses are summed (range 0-63), with higher scores indicating more depressive symptoms. This 21 -item instrument takes less than 10 minutes to administer and is at a $5^{\text {th }}$ grade reading level. Cronbach's alpha of the BDI-II scale is .84 .

\section{Proposed mediators}

Negative thinking: The 45-item Crandall's Cognitions Inventory (CCI) (Crandell \& Chambless, 1986) was used to measure negative thinking. Respondents rate the extent of their agreement with each negative statement on a 5-point Likert scale ranging from "almost never" to "almost always." Total scores range from 34-170, with higher scores indicating more negative thinking. This 45 -item scale takes less than 10 minutes to administer and is at the 6th grade reading level. Cronbach's alphas for the CCI scale are .91 . 
Stress: The 10-item Perceived Stress Scale (PSS) (Cohen, Kamarck, \& Mermelstein, 1983) was used to assess the degree to which the women's lives during the past month were perceived as stressful. The PSS uses a 4-point Likert scale; item responses are summed (range 0-40) with higher scores indicating greater stress. The scale takes less than 5 minutes to administer and is at the $7^{\text {th }}$ grade readability level. Cronbach's alpha for the PSS is .87 .

The 11-item Prenatal Psychosocial Profile (PPP) (Curry Burton, \& Fields, 1998) stress subscale, derived from the Daily Hassles Scale (Kanner, Coyne, Schaefer, \& Lazarus, 1981), was used to assess current stresses/hassles. The PPP uses a 4-point Likert scale; item responses are summed (range 11-44) with higher scores indicating more stress. The scale takes less than 5 minutes to administer and is at a 5th grade reading level. Cronbach's alpha is .76 .

Social-support: The 11-item PPP social support subscale, derived from the Support Behaviors Inventory (Brown, 1986) was used to assess social support from partner and others. The 22-item instrument uses a 6-point Likert scale; item responses are summed (range 11-66), with higher scores indicating more satisfaction. The scale takes less than 5 minutes to administer and is at a 6th grade reading level. Cronbach's alpha for the social support subscale is .93 .

Self-esteem: The 11-item PPP self-esteem subscale, derived from the Rosenberg SelfEsteem Scale (Rosenberg, 1965), was used to assess self-esteem. On this 4-point Likert scale, item responses are summed (range 11-66) with higher scores indicating more selfesteem. The scale takes less than 5 minutes to administer and is at a 6 th grade reading level. Cronbach's alpha of the scale is .75.

\section{Data Analysis}

All analyses were performed using the Statistical Package for the Social Sciences (SPSS) version 22.0 (IBM Corp., 2013). Total scores on stress, negative thinking, self-esteem, social support (combined partner support and support from others), and two measures of antepartum depressive symptoms [the Edinburgh Postnatal Depression Survey (EPDS), and the Beck Depression Inventory-II (BDI-II] were calculated at baseline (T1) and the followup interview 1-month after the intervention ended (T3). All women who completed the interviews at baseline (T1) and at the follow-up interview one month later (T3) were included (completers-only-analysis).

As reported previously (Jesse et al., 2015), an intention-to-treat analysis was not possible because 25 of $72(34.7 \%)$ participants randomized to the intervention did not receive it because of the following reasons: competing work $(n=10)$ or school schedules $(n=3)$, lack of phone service $(n=10)$, fetal demise $(n=1)$, or because they lived too far from the mileage boundaries of the transportation that was provided $(n=1)$. Seven of 72 women $(15 \%)$ assigned to the CBI became ineligible after only 1-2 sessions due to fetal demise (1) or spontaneous abortion $(n=1)$, or premature birth $(n=1)$. Three had a work school schedule conflict and one woman experienced a domestic violence issue that precluded her from staying $(\mathrm{n}=1)$; and one woman completed one of the follow-up interviews but was killed in a 
motor vehicle accident before completing the final follow-up interview. Two of 74 (3\%) participants randomized to treatment-as-usual dropped out before the intervention began because they moved and could not be reached.

The differences between baseline (T1) and 1-month follow-up (T3) scores were computed for each of the targeted measures. Higher change scores indicated reduced stress, more change from negative to positive thinking, enhanced levels of self-esteem, increased social support, and fewer antepartum depressive symptoms.

First, Chi-Square and Fisher's exact tests were performed to examine demographic differences between the treatment and control group and to address and potential issues related to attrition. Then we examined the associations between stress, negative thinking, self-esteem, social support, EPDS and BDI-II by computing Pearson correlations and bootstrapping correlations with 5000 bootstrap samples (Preacher, Rucker, \& Hayes, 2007). The mediation effect was the indirect effect of the intervention on the outcome through the mediators. We wanted to know if the intervention reduced APDS through its effects on these mediators. The PROCESS procedure developed by Hayes (2013) was used to estimate the indirect effects of the intervention using ordinary least squares regression analysis (for continuous outcomes). PROCESS is an SPSS add-on with bootstrap confidence intervals implemented for inferences about indirect effects. The advantage of using bootstrap is that no assumption is made about the distribution of coefficients of the indirect effects.

PROCESS is also easy to implement and the results are straightforward. Thus it is easier to employ than the traditional mediation analysis proposed by Baron and Kenny (1986), in which no inferential test of indirect effect can be obtained. We used $95 \%$ bias-corrected confidence intervals (CIs) based on the 5000 bootstrap samples; with this approach, when the CIs do not include zero, the effect is significant.

\section{Results \\ Sample Characteristics}

Table 1 describes the characteristics of the 110 pregnant women who completed both baseline (T1) and the 1-month follow-up (T3) interviews. Thirty-nine-women were in the $\mathrm{CBI}$ and 71 women were in treatment-as-usual (TAU) at T3. The age of the women ranged from 18-43 years with an average age of $25.59(S D=5.55)$. Over $53 \%$ of the women reported 'single living alone' and $2.7 \%$ reported 'married living alone.' The results showed no significant difference in race distributions between the CBI and TAU groups $(p<0.05)$. More African American women were enrolled in the CBI group (82.1\%) than in the TAU group $(62.0 \%)$. The results showed a significant difference in race distributions between the CBI and TAU groups $(p>0.05)$. Significantly more women dropped out of CBI than TAU, $\chi^{2}(1)=34.29, p<.01$, but no demographic differences were found between women who dropped out and those who stayed in the study ( $p s>0.05$ ). Among the 110 participants who finished both $\mathrm{T} 1$ and $\mathrm{T} 3$ interviews $55.5 \%$ had EPDS scores 10 or greater and $18.3 \%$ reported current major depression at baseline.

Correlations between the mediators and the antepartum depressive symptom outcome measures (EPDS and BDI-II) are shown in Table 2. The bootstrapping correlations, based on 
5000 samples, were very close to the results using the regular Pearson correlation procedure. Biases ranged from -.006 to .006. Therefore, the regular correlation coefficients are reported in Table 2. The correlations were all in a positive direction, which means that from baseline (T1) to the follow-up interview 1 month after the program ended, women who reported experiencing reduced stress and a change from negative thinking to more positive thinking, also reported enhanced levels of self-esteem and fewer antepartum depressive symptoms (EPDS and BDI-II) $(p s<0.05)$. Receiving more social support was significantly associated only with reduced stress $(r=.23, p<0.05)$, not with negative thinking, self-esteem, or the outcome measures (EPDS and BDI-II) $(p s>0.05)$.

PROCESS was used to test the mediation model depicted in Figure 1, with stress, negative thinking, self-esteem, and social support as mediators. Because the results showed a significant difference in the race distribution of the CBI and TAU groups, we controlled for African-American versus Caucasian race in testing the indirect effects of the CBI in reducing antepartum depressive symptoms (EPDS and BDI) through the mediators.

Our first hypothesis was that, controlling for race, women in the culturally tailored cognitive behavioral intervention (CBI) would report more reduction in stress and negative thinking, more enhanced self-esteem, and more increases in social support from baseline (T1) to the 1-month follow-up interview (T3) than those in the treatment-as-usual group. The first step in the mediation analysis was to determine the CBI's effects on the mediators. As can be seen in Table 3, as hypothesized, after controlling for race, women who received the CBI showed greater reductions in levels of stress $(p=.01)$, greater enhancement of self-esteem ( $p$ $=.01)$, and more reductions in negative thinking $(p<.01)$ than those in the TAU group. The results indicated no significant effect of CBI on social support $(p=.49)$. Initially, race was regressed on the model because significantly more African-American women were in the $\mathrm{CBI}$ than in TAU, but race did not play a role in reducing stress, changing negative thinking to more positive thinking, enhancing levels of self-esteem, or increasing social support ( $p \mathrm{~s}$ > $0.05)$.

Our second hypothesis was that, controlling for race, the effects of $\mathrm{CBI}$ in reducing stress, reversing negative thinking, enhancing self-esteem, and increasing social-support would show associations with changes in antepartum depressive symptoms from baseline to 1month follow-up that would be consistent with mediation. The ordinary least squares regression analysis reported in Table 3 indicated that women in the CBI group did not show greater reduction in EDPS and BDI-II scores than the TAU group (EPDS, $p=.55$; BDI-II, $p$ $=.40$ ) controlling for other predictors' effects in the model. However, women who experienced more enhanced self-esteem (EPDS $p<.01$; BDI-II $p=<.01$ ) and more change from negative thinking to more positive thinking ( $\mathrm{p}<.01$; BDI-II, $p=.01$ ) were also more likely to report decreased levels of antepartum depressive symptoms (EPDS and BDI-II). Reducing stress significantly decreased BDI-II scores $(p<.01)$, but not EPDS scores $(p=$. 07).

The results showed no direct effects of the CBI on reducing antepartum depression symptoms without mediators in the model (Coefficient $\mathrm{EPDS}=.44,95 \% \mathrm{CI}=-1.02-1.90$ and Coefficient $\left._{\text {BDI-II }}=-1.02,95 \% \mathrm{CI}=-3.43-1.38\right)$. However, bias-corrected confidence 
intervals for the total indirect effects of the CBI on reducing EPDS and BDI-II scores through mediators based on 5000 bootstrap samples did not include zero (Coefficient ${ }_{\text {EPDS }}=$ $1.79,95 \% \mathrm{CI}=.54-3.17$ and Coefficient $\mathrm{BDI}-\mathrm{II}=3.87,95 \% \mathrm{CI}=1.42-7.06)$. Bias-corrected confidence intervals based on 5000 bootstrap samples also did not include zero for stress (Coefficient ${ }_{\text {EPDS }}=.46,95 \% \mathrm{CI}=.19-1.45$ and Coefficient $\mathrm{BDI}-\mathrm{II}=1.56,95 \% \mathrm{CI}=.40$ 3.51), self-esteem (Coefficient ${ }_{\mathrm{EPDS}}=.62,95 \% \mathrm{CI}=.10-1.47$ and Coefficient $\mathrm{BDI}-\mathrm{II}=1.15$, $95 \% \mathrm{CI}=.27-2.60)$, and negative thinking (Coefficient $\mathrm{EPDS}=.74,95 \% \mathrm{CI}=.20-1.71$ and Coefficient $\mathrm{BDI}-\mathrm{II}=1.11,95 \% \mathrm{CI}=.20-2.88$ ). In other words, over time, reduced stress, enhanced self-esteem and change from negative thinking to more positive thinking were significantly associated with decreased antepartum depressive symptoms (EPDS and BDI-II) for women in the CBI but not for those in TAU. However, the results did not show that increased levels of social-support reduced EPDS and BDI-II scores more for women in the $\mathrm{CBI}$ than for those in TAU (Coefficient $\mathrm{EPDS}=-.03,95 \% \mathrm{CI}=-.42-.09$ and Coefficient $\mathrm{BDI}-\mathrm{II}=.06,95 \% \mathrm{CI}=-.15-.77)$.

\section{Discussion}

This analysis examined the mechanism of the effects of Insight-Plus, a theoretically based and culturally tailored cognitive behavioral intervention (CBI) to reduce antepartum depressive symptoms (APDS) in rural low-income and minority women. Analysis indicated that women in the CBI group experienced significantly more stress reduction, more change from negative thinking to more positive thinking, and more enhanced self-esteem, than women in TAU. However, CBI had no effect on social-support.

In an earlier randomized clinical trial with non-pregnant women ( $\mathrm{n}=136)$, Peden, Rayens, Hall, \& Grant (2005) delivered a CBI for low-income women based on Gordon et al.'s (1988) cognitive-behavioral program, similar to that adapted for rural pregnant low-income and minority women in our study. Crandell's Cognition Inventory to measure negative thinking used in Peden et al.'s study (2005) was also included in our study. The women in their study showed more reduction in stress and negative thinking, and fewer depressive symptoms than those in the control group; however, self-esteem and social support were not included in their study. Similarly, in another RCT (Allard-VanDam, Hosman, \& Hoogduin, 2003) with non-pregnant adults living in the Netherlands, the authors reported that a cognitive-behavioral psycho-education intervention based on the "Coping with Depression" course was effective in reducing depressive symptoms and negative thinking, increasing pleasant life activities, enhancing self-esteem, and increasing frequency of receiving social support for participants with subclinical depression. Conversely, in another RCT (Urizar \& Munoz, 2011), Spanish speaking low-income pregnant mothers $(n=126)$ in a prenatal cognitive behavioral stress management (CBSM) intervention group showed significantly higher levels of stress and more negative affect at 6 months postpartum than women in TAU.

In our study, increases in social support produced by the CBI were not associated with reductions in APDS, but increased social support was significantly associated with reduced levels of stress. In a systematic review, Lancaster, Gold, Flynn, Marcus, and Davis (2010) reported that higher levels of stress and social support were associated with fewer depressive symptoms in pregnancy. Activities that enhance self-esteem and social-support may also 
buffer the negative impact of stress and thus potentially reverse the progression of depressive symptoms in rural pregnant women (Jesse et al., 2014).

In our study, 55.5\% of the women were at risk for depression and $18.3 \%$ of those reported current major depression. Because an EPDS score of 4 or greater was an inclusion criterion for eligibility for our study, the percentage of women experiencing APDS and APD may have been higher than the average reported in other studies (Gaynes et al., 2005; Jesse et al., 2014). Only $16 \%$ of the women in our study were married, $27 \%$ were single living with a partner; over half were single or married living alone without emotional or financial support from a partner. Many had partners who were unsupportive or absent and some could not identify any support person to invite to a CBI session. However, women commented positively about the support they received from other members enrolled in the group sessions and from the study team members. The findings from this and other studies highlight the clinical importance of continuing to include and refine social support activities to reduce stress for women at risk of depression, particularly if they are single and without partner support. While a CBI cannot compensate for lack of partner or other support in women's lives, the women can use skill-building activities to help build a healthier support network, to communicate better with existing support persons, and to learn to ask for what they need/want.

We found that over time women who experienced enhanced levels of self-esteem and greater change from negative thinking to positive thinking were more likely to report decreased levels of APDS (EPDS and BDI-II). Furthermore, reducing stress significantly decreased BDI-II scores, though not EPDS scores.

Changing negative thinking to positive thinking showed the strongest associations with reductions in APDS, as measured by the EPDS, and reduction in stress showed the strongest association with reduction in APDS as measured by the BDI-II. Others have reported similar indirect effects changing negative thinking to more positive thinking in reducing depressive symptoms in university students (Allard-VanDam et al., 2003) and in minority low-income adults in primary care (Urizar \& Munoz, 2011).

The low-income and minority women in this study had many stressors during pregnancy, including financial difficulties, living in poverty, and death of a loved one. The study findings highlight the importance of activities that reduce stress, reduce negative thinking, and enhance self-esteem in order to reduce antepartum depressive symptoms in rural lowincome women. While interventions such as this CBI cannot eliminate poverty directly, pregnant low-income women can use the activities of the CBI to seek alternatives to their present responses to stressors and negative thinking.

\section{Limitations}

Although our findings are promising, this second analysis of primary data from a randomized controlled clinical trial has the limitations usually found in cross-sectional research. While the findings suggest that reducing stress, enhancing self-esteem, and changing cognitions may be associated with change in depressive symptoms in a manner consistent with causal mediation, they are not sufficient to rule out other interpretations. 
Although changes in mediators and changes in outcome were correlated over time for some of our mediators, the associations could also be a consequence of reverse causality (treatment induced reductions in antepartum depressive symptoms drove changes in stress and cognition) or third variable causality (neither mediator nor outcome had a causal impact on the other, but changes in each were associated because the CBI had a causal impact on each mediator). However, viewed in the context of our theoretical framework and previous research, our findings increase confidence that the mediators we examined played a causal role. To strengthen mediation analysis, future researchers need to assess mediator variables and dependent variables at different time points over the course of the CBI. However, this has to be balanced with not over-burdening low-income pregnant women. Because of the physical/emotional needs of these women, they may tire easily, especially if they are experiencing depressive symptoms and their consequences.

Finally, similar to other RCTs with this population (Lara, Navarro, \& Navarrete, 2009), women enrolled in the CBI had a higher rate of attrition and loss to follow-up than women in the TAU group, despite offering transportation, childcare and multiple methods of contact. This confirms what others (Grote et al., 2009; Lara et al., 2009) have found, that it is challenging to recruit and retain low-income and minority pregnant women in intervention research who are experiencing antepartum depressive symptoms or depression. Future RCTs with this population should consider a two-step recruitment and engagement process similar to that described in a RCT of an interpersonal psychotherapy for perinatal depression in urban low-income women (Grote et al., 2009).

\section{Conclusions}

This analysis is the first to examine the mediators underlying the beneficial effects of a CBI in rural, low-income, minority women. Despite its limitations, the study increases our understanding of the interrelationships among the concepts in Jesse's theory (Jesse, Kim, Herndon, 2015). Our findings suggest that the CBI may reduce antepartum depressive symptoms through reducing stress, changing negative thinking to more positive thinking, and enhancing self-esteem. These findings are not sufficient to rule out other alternative interpretations, but they are consistent with the notion of mediation. The findings thus provide a better understanding of the mechanisms underlying CBI's beneficial effects on APDS in rural low-income and minority women. Based on these findings, it may be important for Master's level mental health nurses and other mental health professionals to offer cognitive-behavioral therapies to reverse the progression of antepartum depressive symptoms that include activities to reduce stress, change negative cognitions, and enhance self-esteem.

\section{References}

Accortt EE, Schetter CD. Pregnant women screening positive for depressive symptoms at 24-28 weeks may have increased risk of preterm birth but more precise research is needed. Evidence-Based Nursing. 2014; 17(1):11-12. http://doi.org/10.1136/eb-2012-101164. [PubMed: 23525078]

Allard-VanDam E, Hosman CMH, Hoogduin CAL. The coping with depression course: Short-term outcomes and mediation effects of a randomized controlled trial in the treatment of subclinical depression. Behavioral Therapy. 2003; 34:381-96. DOI: 10.1016/S0005-7894(03)80007-2 
Baron RM, Kenny DA. The moderator-mediator variable distinction in social psychological research: Conceptual, strategic and statistical considerations. Journal of Personality and Social Psychology. 1986; 51:1173-1182. [PubMed: 3806354]

Beck, AT. Depression: Clinical experimental and theoretical aspects. New York: Harper Row; 1967.

Beck, AT. Cognitive therapy and the emotional disorders. New York: International Universities Press; 1976.

Beck, AT.; Brown, G.; Steer, RA. Beck Depression Inventory II Manual. San Antonia, TX: The Psychological Corporation; 1996.

Brown MA. Social support during pregnancy: A unidimensional or multidimensional construct? Nursing Research. 1986; 35(1):4-9. DOI: 10.1097/00006199-198601000-00002 [PubMed: 3632846]

Cohen S, Kamarck T, Mermelstein R. A global measure of perceived stress. Journal of Health \& Social Behavior. 1983; 24:385-396. [PubMed: 6668417]

Cox JL, Holden IM, Sagovsky R. Detection of postnatal depression: Development of the 10-item Edinburgh Postnatal Depression Scale. British Journal of Psychiatry. 1987; 150:782-786. [PubMed: 3651732]

Crandell CJ, Chambless DL. The validation of an inventory for measuring depressive thoughts: The Crandell Cognitions Inventory. Behaviour Research \& Therapy. 1986; 24(4):403-11. DOI: 10.1016/0005-7967(86)90005-7 [PubMed: 3741306]

Curry MA, Burton D, Fields J. The prenatal psychosocial profile: a research and clinical tool. Research in Nursing \& Health. 1998; 21:211-219. DOI: 10.1002/(SICI)1098-240X(199806)21:3<211:AIDNUR4>3.0.CO;2-K [PubMed: 9609506]

Fellenzer JL, Cibua DA. Intendedness of pregnancy and other predictive factors for symptoms of prenatal depression in a population-based study. Maternal Child Health Journal. 2014; 18(10): 2426-36. DOI: 10.1007/s10995-014-1481-4 [PubMed: 24752314]

Gaynes, BN.; Gavin, N.; Meltzer-Brody, S.; Lohr, KN.; Swinson, T.; Gartlehner, G.; Miller, WC. Evidence Report/Technology Assessment No. 119. Rockville, MD: Agency for Healthcare Research and Quality; 2005. Perinatal depression: Prevalence, screening accuracy, and screening Outcomes. AHRQ Publication No. 05-E006-2(Prepared by the RTI-University of North Carolina Evidence-based Practice Center, under Contract No. 290-02-0016.)

Giurgescu C, Engeland CG, Templin TN. Symptoms of depression predict negative birth outcomes in African-American women: A pilot study. Journal of Midwifery \& Women's Health. 2015; 60(5): 570-577.

Gordon VC, Matwychuk AK, Sachs EG, Canedy BH. A 3-year follow-up of a cognitive-behavioral therapy intervention. Archives of Psychiatric Nursing. 1988; 2:218-26. [PubMed: 3178300]

Gourounti K, Karapanou V, Karpathiotaki N, Vaslamatzis G. Anxiety and depression of high risk pregnant women hospitalized in two public hospital settings in Greece. International Archives of Medicine. 2015; 8(25)doi: 10.3823/1624

Grote NK, Swartz H, Geibel S, Zuckoff A, Houck P, Frank E. A randomized controlled trial of culturally relevant, brief interpersonal psychotherapy for perinatal depression. Psychiatric Services. 2009; 60(3):313-321. [PubMed: 19252043]

Hayes, AF. Introduction to mediation, moderation, and conditional process analysis: A regressionbased approach. Guilford Press; 2013.

IBM Corp. IBM SPSS Statistics for Windows, Version 22.0. Armonk, NY: IBM Corp; Released 2013

Jallo N, Elswick RK, Kinser P, Masho S, Price K, Svikis DS. Prevalence and predictors of depressive symptoms in pregnant African-American women. Issues in Mental Health Nursing. 2015; 36(11): 860-869. DOI: 10.3109/01612840.2015.1048014 [PubMed: 26631857]

Jesse DE, Dolbier CL, Blanchard A. Barriers to seeking help and treatment suggestions for prenatal depressive symptoms: Focus groups with rural low-income women. Issues in Mental Health Nursing. 2008; 29(1):3-19. DOI: 10.1080/01612840701748664 [PubMed: 18214775]

Jesse DE, Gaynes BN, Feldhousen EB, Newton ER, Bunch S, Hollon SD. Performance of a Culturally Tailored Cognitive-Behavioral Intervention Integrated in a Public Health Setting to Reduce Risk of Antepartum Depression: A Randomized Controlled Trial. Journal of Midwifery \& Women's Health. 2015; 60(5):578-592. 
Jesse DE, Kim H, Herndon C. Social support and self-esteem: Mediators between perceived stress and depressive symptoms in rural pregnant women. Research in Nursing \& Health. 2014; 37(3):24152. DOI: 10.1002/nur.21600 [PubMed: 24797585]

Jesse DE, Swanson M. Risks and resources associated with antepartum risk for depression among rural southern women. Nursing Research. 2007; 56(6):378-386. DOI: 10.1097/01.NNR. 0000299856.98170.19 [PubMed: 18004184]

Kanner AD, Coyne JC, Schaefer C, Lazarus RS. Comparison of two modes of stress measurement: Daily hassles and uplifts versus major life events. Journal of Behavioral Medicine. 1981; 4(1):139. DOI: 10.1007/BF00844845 [PubMed: 7288876]

Ko JY, Farr SL, Dietz PM, Robbins CL. Depression and treatment among U.S. pregnant and nonpregnant women of reproductive age, 2005-2009. Journal of Women's Health. 2012; 21(8): 830-836. DOI: $10.1089 /$ jwh.2011.3466

Lancaster C, Gold K, Flynn H, Marcus S, Davis M. Risk factors for depressive symptoms during pregnancy: A systematic review. American Journal of Obstetrics \& Gynecology. 2010; 202(1):514. DOI: 10.1016/j.ajog.2009.09.007 [PubMed: 20096252]

Lara MA, Navarro C, Navarrete L. Outcome results of a psychoeducational intervention in pregnancy to prevent PPD: A randomized controlled trial. Journal of Affective Disorders. 2009; 122:109-117.

Linde K, Sigterman K, Kriston L, Rucker G, Jamil S, Meissner K, Schneider A. Effectiveness of psychological treatments for depressive disorders in primary care: Systematic review and metaanalysis. Annals of Family Medicine. 2015; 13(1):56-68. DOI: 10.1370/afm.1719 [PubMed: 25583894]

Palladino CL, Singh V, Campbell J, Flynn H, Gold K. Homicide and suicide during the perinatal period: Findings from the national violent death reporting system. Obstetric Anesthesia Digest. 2011; 32(4):217-218. DOI: 10.1097/01.aoa.0000422697.74027.32

Peden AR, Rayens MK, Hall LA, Grant E. Testing an intervention to reduce negative thinking, depressive symptoms, and chronic stressors in low-income single mothers. Journal of Nursing Scholarship. 2005; 37(3):268-74. DOI: 10.1111/j.1547-5069.2005.00046.x [PubMed: 16235869]

Preacher KJ, Rucker DD, Hayes AF. Addressing moderated mediation hypotheses: Theory, methods, and prescriptions. Multivariate Behavioral Research. 2007; 42(1):185-227. DOI: 10.1080/00273170701341316 [PubMed: 26821081]

Rosenberg, M. Society and the adolescent self-image. Princeton, NJ: Princeton University Press; 1965.

Smedberg J, Lupatelli A, Mardby AC, Overland S, Nordeng H. The relationship between maternal depression and smoking cessation during pregnancy-a cross-sectional study of pregnant women from 15 European countries. Archives of Women's Mental Health. 2015; 18(1):73-84. DOI: 10.1007/s00737-014-0470-3

Tomita A, Labys CA, Burns JK. Depressive Symptoms Prior to Pregnancy and Infant Low Birth Weight in South Africa. Maternal Child Health Journal. 2015; 19(10):2179-86. DOI: 10.1007/ s10995-015-1732-z [PubMed: 25673370]

Urizar GG, Munoz RF. Impact of a prenatal cognitive-behavioral stress management intervention on salivary cortisol levels in low-income mothers and their infants. Psychoneuroendocrinology. 2011; 36:1480-1494. DOI: 10.1016/j.psyneuen.2011.04.002 [PubMed: 21641117]

Vesga-López O, Blanco C, Keyes K, Olfson M, Grant BF, Hasin DS. Psychiatric disorders in pregnant and postpartum women in the United States. Archives of General Psychiatry. 2008; 65(7):805815. http://dx.doi.org/10.1001/archpsyc.65.7.805. [PubMed: 18606953]

Witt WP, Wisk LE, Cheng ER, Hampton JM, Creswell PD, Hagen EW, DeLeire T. Poor prepregnancy and antepartum mental health predicts postpartum mental health problems among US women: a nationally representative population-based study. Women's Health Issues. 2011; 21(4):304-313. [PubMed: 21349740] 


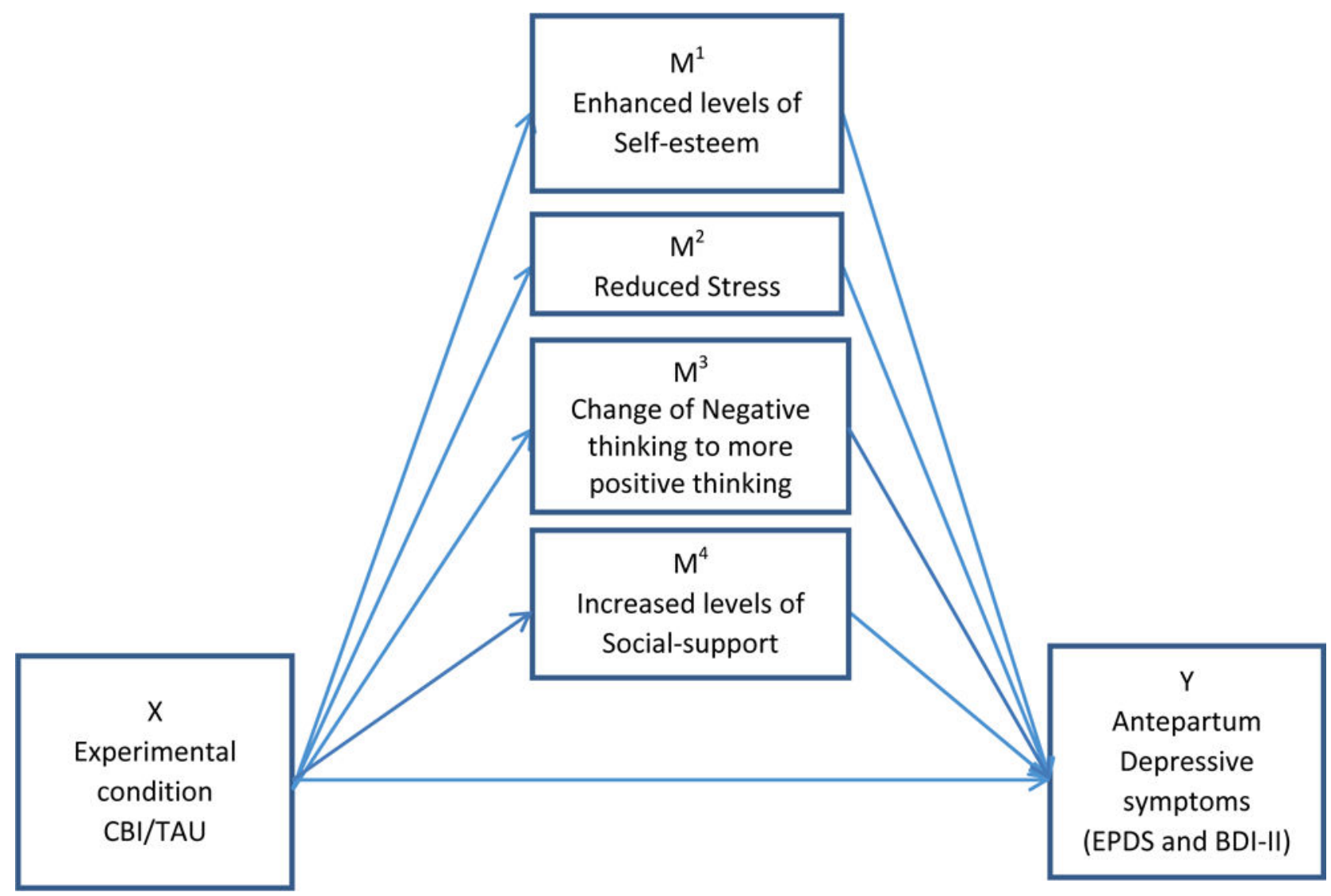

Figure 1.

A mediation model showing the mechanism of the CBI effect. In this figure, $\mathrm{X}$ represents the independent variable [Cognitive Behavioral Intervention (CBI) versus Treatment-asUsual (TAU)] and Y, the dependent variable, measures antepartum depressive symptoms [Edinburgh Postnatal Depression Scale (EPDS) and the Beck Depression Inventory (BDI_II)]. Finally, M1-M4 represents the proposed mediator variables 


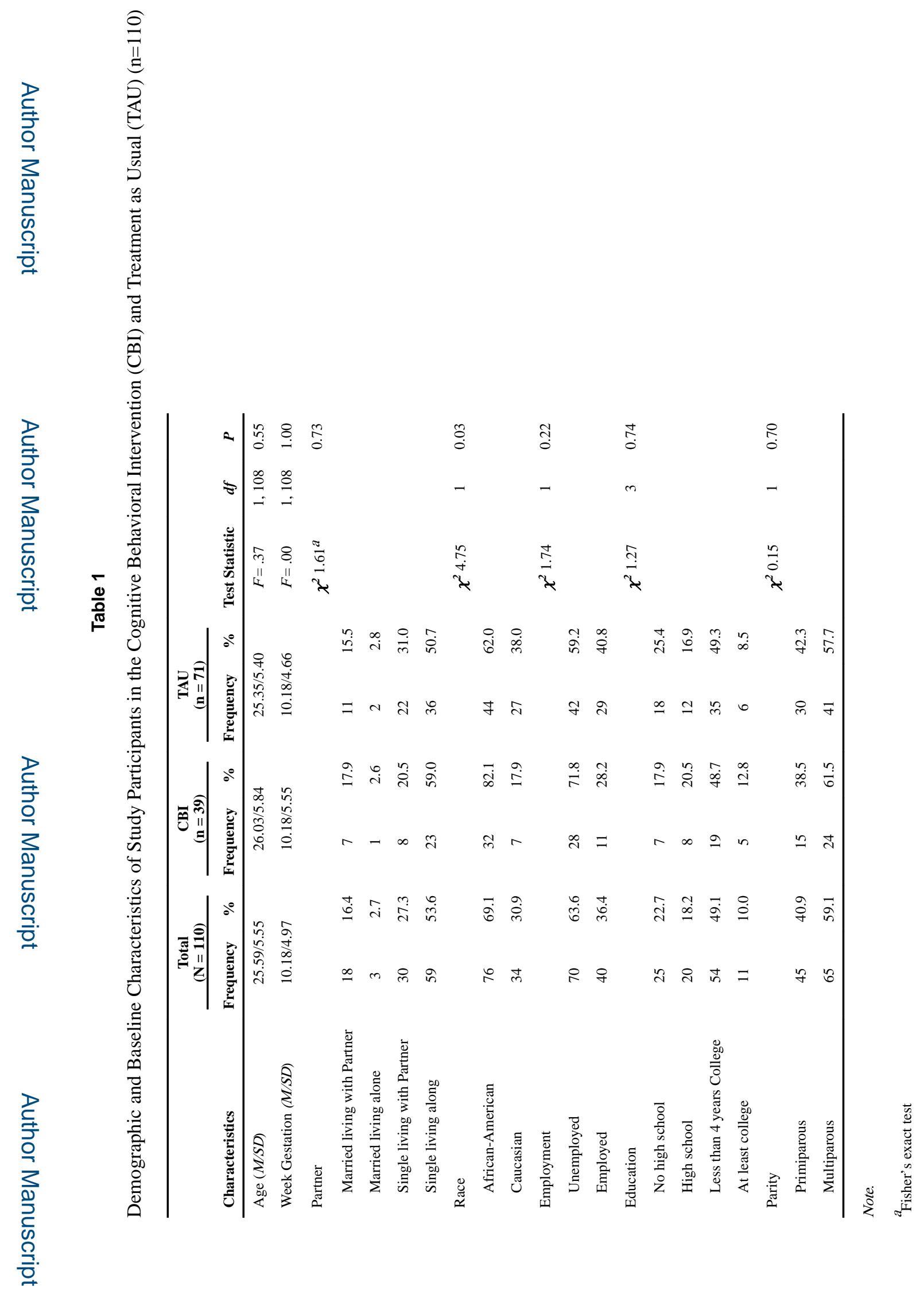

Issues Ment Health Nurs. Author manuscript; available in PMC 2017 November 01. 




Issues Ment Health Nurs. Author manuscript; available in PMC 2017 November 01 


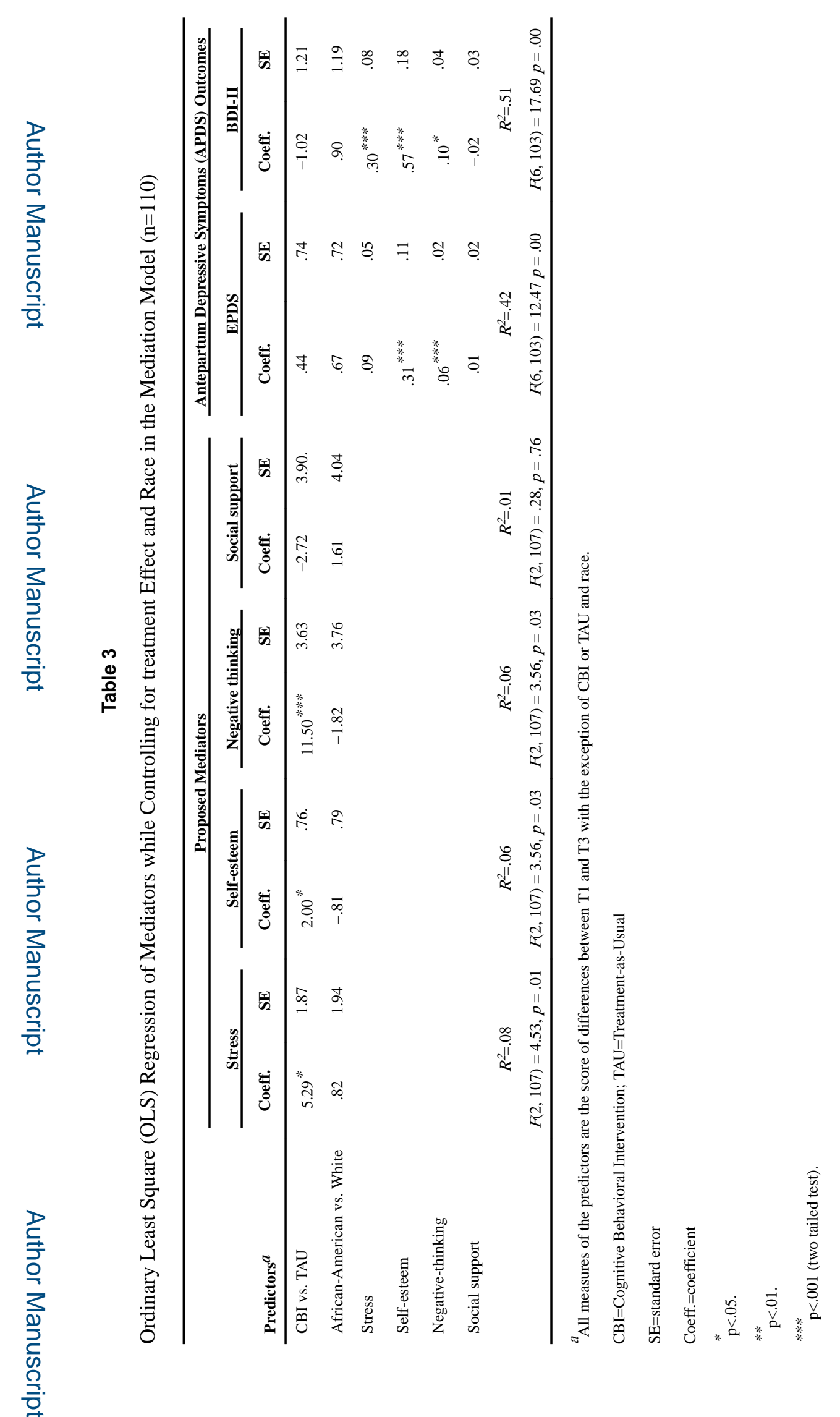

Issues Ment Health Nurs. Author manuscript; available in PMC 2017 November 01. 